\title{
Aclimatación en cautiverio del sargo Archosargus probatocephalus (Perciformes: Sparidae)
}

\section{Acclimation in captivity of the sheepshead Archosargus probatocephalus (Perciformes: Sparidae)}

María de la Luz Merino-Contreras ${ }^{1,2 *}$, Froylán Sánchez-Morales ${ }^{2}$, María de Lourdes Jiménez-Badillo ${ }^{1}$, Carlos Alfonso Álvarez-González ${ }^{3}$, César Gabriel Meiners-Mandujano ${ }^{1}$, Emyr Saúl Peña-Marín ${ }^{3}$

${ }^{1}$ Instituto de Ciencias Marinas y Pesquerías, Universidad Veracruzana. Calle Hidalgo 617, Col. Río Jamapa, CP. 94290. Boca del Río, Veracruz, México.

${ }^{2}$ Instituto Tecnológico de Boca del Río. Departamento de Ciencias del mar. Carretera Federal Veracruz-Cordoba km 12. CP. 94290. Boca del Rio, Veracruz, México.

${ }^{3}$ Cátedra-CONACyT. Universidad Juárez Autónoma de Tabasco. Km. 0.5 carretera Villahermosa-Cárdenas entronque Bosques de Saloya, CP. 86160. Villahermosa, Tabasco, México.

*Autor de correspondencia: madelaluz.merino020@gmail.com.

Artículo científico recibido: 18 de enero de 2018 aceptado: 02 de junio de 2018

RESUMEN. El sargo (Archosargus probatocephalus) es una especie prioritaria para el desarrollo de la maricultura en el sureste mexicano. El objetivo del estudio fue reproducir en cautiverio dos lotes de sargos, uno en cautiverio por 12 meses y otro de reproductores silvestres. El lote en cautiverio se formó por 36 organismos, en un sistema de recirculación de agua con salinidad de 10 psu, a los que se les determinó el incremento en longitud y peso cada mes, además se promovió la madurez sexual incrementando de forma gradual la salinidad del sistema hasta 35 psu, para luego inducir el desove con una inyección intramuscular de $500 \mathrm{Ul} \mathrm{kg}^{-1}$ de hormona gonadotropina coriónica y sérica. Por otra parte, se colectaron 15 reproductores silvestres de la laguna de Alvarado Veracruz, induciéndolos al desove de la misma forma. El incremento promedio en longitud de los organismos acondicionados al cautiverio fue de $0.064 \pm 0.02 \mathrm{~cm} \mathrm{~d}^{-1}$ con peso promedio de $1.97 \pm 0.4 \mathrm{~g} \mathrm{~d}^{-1}$, madurando sexualmente en el mes de enero. Ambos lotes desovaron, los huevos obtenidos tuvieron un diámetro promedio de $817.3 \pm 20.9 \mu \mathrm{m}$, volumen del saco vitelino promedio de $243 \pm 11 \mathrm{~nL}$, diámetro promedio de la gota de aceite de $203.5 \pm 16.5 \mu \mathrm{m}$ y longitud notocordal promedio de los eleuteroembriones de $1.65 \pm 0.25 \mathrm{~mm}$. Es posible lograr la maduración en cautiverio del sargo, así como, obtener larvas de calidad a partir de la inducción de reproductores en cautiverio y silvestres en la época reproductiva.

Palabras clave: Aclimatación, maduración sexual, reproducción, desove, larvas

ABSTRACT. The sheepshead (Archosargus probatocephalus) is a priority species for mariculture development in southeastern Mexico. The objective of the study was to reproduce in captivity two sheepshead batches, one in captivity for 12 months and another of wild breeders. The batch in captivity was formed by 36 organisms, in a water recirculation system with salinity of $10 \mathrm{psu}$, with the increase in their length and weight determined each month; in addition, sexual maturity was promoted by gradually increasing the salinity of the system up to $35 \mathrm{psu}$, after which spawning was induced with an intramuscular injection of $500 \mathrm{Ul} \mathrm{kg}^{-1}$ of chorionic and serum gonadotropin hormone. On the other hand, 15 wild breeders were collected from the Alvarado Veracruz lagoon, inducing them to spawn in the same way. The average increase in length of the organisms conditioned to captivity was $0.064 \pm 0.02 \mathrm{~cm} \mathrm{~d}^{-1}$ with average weight of $1.97 \pm 0.4 \mathrm{~g} \mathrm{~d}^{-1}$, sexually maturing in the month of January. Both batches spawned; the eggs obtained had an average diameter of $817.3 \pm 20.9 \mu \mathrm{m}$, average yolk sac volume of $243 \pm 11 \mathrm{~nL}$, average oil drop diameter of $203.5 \pm 16.5 \mu \mathrm{m}$ and average notochordal length of the eleuteroembryos of $1.65 \pm 0.25 \mathrm{~mm}$. It is possible to achieve maturation of the sheepshead in captivity, as well as obtain quality larvae from the induction of breeders in captivity and wild ones in the reproductive season.

Key words: Acclimation, sexual maturation, reproduction, spawning, larvae 


\section{INTRODUCCIÓN}

El sargo (Archosargus probatocephalus, Walbaum 1792), es un pez marino costero subtropical que ingresa a las aguas salobres, se distribuye en las costas del Océano Atlántico occidental (Bester y Robins 2005), desde Nueva Escocia en Canadá hasta el Golfo de México, con registros en Honduras y Brasil (GSMFC 2006). Es un componente importante de las pesquerías recreativas deportivas y comerciales a lo largo de la costa Atlántica de EUA y Golfo de México (McDonough et al. 2011). El sargo pertenece a la familia Sparidae, una de las más cultivadas en el mundo, con representantes importantes como la brema roja (Pagrus major) en Japón y Taiwan (Okuzawa 2015) y la dorada (Sparus aurata) en el Mediterráneo (APROMAR 2017). El sargo tiene potencial en la acuacultura, debido a su gran adaptabilidad al cautiverio, amplio espectro de alimentación, soporta las condiciones de hacinamiento, se ha logrado el desove y la producción de crías en laboratorio, puede pesar hasta $14 \mathrm{~kg}$, alcanzar una longitud máxima de $80 \mathrm{~cm}$, y su carne es de excelente calidad (Tucker 1998, 2004).

Se tienen reportes de que desova en los arrecifes cercanos a las costas del Atlántico medio y sur de los Estados Unidos y en los estuarios del Golfo de México, a finales del invierno y principios de primavera (GSMFC 2006). Mientras que McDonough et al. (2011) reportaron en Carolina del Sur, con una época de reproducción de enero a abril. La talla de primera madurez para $A$. probatocephalus es de $35 \mathrm{~cm}$ en las costas de los Estados Unidos (Wenner 2004). El promedio del diámetro del ovocito maduro es de $400 \mu \mathrm{m}$, tamaño en que se recomienda el uso de 1000 a $2000 \mathrm{UI} \mathrm{kg}^{-1}$ de hormona gonadotropina coriónica humana para inducir el desove (Tucker 1998). Esta especie es considerada gonocórica y desovadora parcial, madura generalmente a los dos años de edad (Wenner 2004, Tucker 2004). La frecuencia de desove se estima, desde una vez al día por siete días, hasta una vez cada $20 \mathrm{~d}$ (Munyandorero et al. 2006). Al respecto Mc Donough et al. (2011) señalan que la fecundidad parcial promedio es variable, y se correlaciona con la longitud patrón, el peso y la edad. En hembras capturadas en aguas costeras, es de 1100 a 40000 ovocitos por desove, mientras que, en hembras capturadas en alta mar, es de 14000 a 250000 ovocitos (GSMFC 2006). En cautiverio Tucker y Barbera (1987) obtuvieron 15000 huevos desovados de forma manual, con individuos con peso promedio de $378 \mathrm{~g}$. Al respecto Tucker y Alshuth (1997) reportan que los huevos son pelágicos de $0.82 \mathrm{~mm}$ de diámetro promedio, poseen una gota de aceite que mide $214 \pm 27 \mu \mathrm{m}$, eclosionan $28 \mathrm{~h}$ después de la fecundación, a temperatura de $23{ }^{\circ} \mathrm{C}$. Mientras que Tucker y Barbera (1987) reportan una tasa de fertilización de 59\% y eclosión del $86 \%$, en tanto que Tucker (1998, 2004) reportan una sobrevivencia larval al séptimo día después de la eclosión del $62 \%$. La longitud de la larva al eclosionar es de $1.65 \pm 0.15 \mathrm{~mm}$ (Tucker 2004), a los tres días después de la eclosión los ojos y el sistema digestivo son funcionales y comienza su alimentación exógena, al cuarto día post-eclosión, el vitelo y la gota de aceite se agotan y la larva se alimenta por ella misma (Tucker y Alshuth 1997).

Aunque pareciera que se conocen las técnicas de reproducción de la especie, el manejo de reproductores acondicionados al cautiverio no se ha realizado de manera exitosa (Tucker 1998, 2004), siendo esto esencial para la obtención de larvas de manera eficiente, por lo que el objetivo del presente estudio fue evaluar la reproducción de dos lotes de sargos, uno mantenido en cautiverio por un año y otro silvestre, logrando el desove en ambos lotes.

\section{MATERIALES Y MÉTODOS}

\section{Lote acondicionado y madurado sexualmente en cautiverio}

Se capturaron 45 juveniles de sargo ( $A$. probatocephalus) en el mes de febrero del 2014 en la laguna de Alvarado, Veracruz, México, con peso promedio de $100 \pm 50 \mathrm{~g}$, para luego transportarlos en contenedores con aireación al Laboratorio de Acuacultura del Instituto Tecnológico de Boca del Río, donde se les dio un baño profiláctico con peróxido de hidrógeno a $400 \mathrm{ppm}$ por $30 \mathrm{~min}$ y agua dulce, para luego colocarlos en un sistemas de re- 
circulación por un año, el sistema de recirculación estuvo conformado por dos tinas de fibra de vidrio con capacidad de $23 \mathrm{~m} 3$, ensambladas con un filtro biológico de $7 \mathrm{~m}^{3}$, los peces se colocaron a densidad de un 1 organismo por $\mathrm{m}^{3}$, la calidad del agua del sistema se registró con una sonda multiparamétrica (Hanna HI 9828), salinidad $10 \pm 1$ psu, temperatura de $25.5 \pm 3^{\circ} \mathrm{C}$, oxígeno disuelto de $4.5 \pm 0.3 \mathrm{mg}$ $\mathrm{L}^{-1}$ y amonio total de $0.1 \mathrm{mgL}^{-1}$. Se registró la supervivencia (\%) de los organismos, la longitud furcal (LF) en $\mathrm{cm}$ con un ictiómetro y el peso en g con una báscula digital Ohaus ${ }^{\circledR}(0.01 \mathrm{~g})$.

Los organismos se aclimataron al cautiverio por medio de la administración inicial al libitum de ostiones y pescado frescos, por cinco días y gradualmente se cambió a alimento balanceado semihúmedo con $45 \%$ de proteína y $12 \%$ de lípidos, conformado por alimento balanceado de iniciación para tilapia (Purina), filete de pescado fresco, calamar, lecitina de soya, aceite de pescado, alginato de sodio y mezcla de vitaminas y minerales. Al octavo mes de su acondicionamiento, se indujo la madurez sexual por medio del incremento de forma gradual de la salinidad del sistema, incrementando 5 psu cada semana, hasta 35 psu.

\section{Lote de organismos silvestres}

En el mes de febrero de 2015 se capturaron en la laguna de Alvarado, Veracruz, México, 15 organismos, 5 hembras y 10 machos con peso promedio de $600 \pm 200 \mathrm{~g}$ y $26 \pm 2 \mathrm{~cm}$ de LF, para formar el lote de organismos silvestres. Los cuales se transportaron al Laboratorio de Acuacultura del Instituto Tecnológico de Boca del Río, para su revisión, determinar el grado de madurez sexual por medio de biopsias de la gónada y luego observar las muestras al microscopio compuesto (Carl Zeiss, Primo Star) registrando en el caso de las hembras, la morfología y el diámetro de los ovocitos, y en los machos la movilidad de los espermas, la apariencia y fluidez del semen.

Con los peces maduros sexualmente en ambos lotes; machos con semen fluido y hembras con ovocitos en vitelogénesis avanzada con más de 400 $\mu \mathrm{m}$ de diámetro, se registró su peso y se indujo el desove con la aplicación intramuscular de $500 \mathrm{UI}$ $\mathrm{kg}^{-1}$ de hormona gonadotropina coriónica y sérica de uso veterinario (PG 600 Intervet). Para despues colocar los reproductores en una proporción de dos hembras y cuatro machos por tina, en un sistema cerrado con dos tinas de fibra de vidrio de $1.2 \mathrm{~m}^{3}$, acopladas a un reservorio de sedimentación y filtro biológico, con capacidad de 620 L. Se registró el tiempo en que los organismos desovaron después de la inyección de la hormona, y se evaluó la viabilidad del desove, colocando los huevos en una probeta de cristal de $1000 \mathrm{~mL}$, para luego esperar a que se separaran los huevos viables en la parte superficial y los inviables en el fondo. Los huevos viables de cada desove se colocaron en una incubadora invertida de 100 L hasta su eclosión. Los eleuteroembriones obtenidos se mantuvieron en peceras de vidrio de 60 $\mathrm{L}$ a densidad de 100 organismos $\mathrm{L}^{-1}$ con aireación suave, adicionando la microalga Nannochloropsis oculata $100000 \mathrm{cel} \mathrm{mL}^{-1}$. La alimentación exógena inició a partir del segundo día después de la eclosión (DDE) suministrando el rotífero Brachionus plicatilis morfotipo $\mathrm{L}$ ( 5 rotíferos $\mathrm{mL}^{-1}$ ). Los parámetros de la calidad del agua se determinaron con una sonda multiparamétrica (Hanna $\mathrm{HI}$ 9828): salinidad $35 \pm 1$ psu, temperatura de 21.5 $\pm 0.5{ }^{\circ} \mathrm{C}$, oxígeno disuelto de $4.2 \pm 0.3 \mathrm{mg} \mathrm{L}^{-1} \mathrm{y}$ amonio total de $0.1 \mathrm{mgL}^{-1}$.

Para evaluar la calidad de los desoves, se registró el diámetro de la gota de aceite, el volumen del saco vitelino y el tamaño promedio de los huevos y eleuteroembriones, para lo cual se utilizó un microscopio compuesto (Carl Zeiss, Primo Star) con micrómetro ocular con aumento de 4x. Durante los nueve días que sobrevivieron las larvas (DDE), se evaluó el crecimiento en longitud notocordal (LN) $(\mathrm{mm})$ y la tasa de supervivencia $\left(\% \mathrm{~d}^{-1}\right)$.

\section{Análisis estadístico}

Se verifico la normalidad (KolmogorovSmirnov) y homocedasticidad (Levene) del diámetro de la gota de aceite, el volumen del saco vitelino y el tamaño promedio de los huevos y eleuteroembriones recién eclosionados. El análisis paramétrico de comparación entre grupos se determinó con la 
prueba de $t$ de Student de muestras independientes $(p<0.05)$, mientras que el porcentaje de eclosión y supervivencia de las larvas entre los grupos se analizó con la prueba de $\mathrm{Chi}^{2}$ a partir de la transformación arcoseno. Todos los análisis estadísticos se realizaron con el programa Statistic v 7.1.

\section{RESULTADOS}

\section{Lote acondicionado y maduración sexual en cautiverio}

El pre-acondicionamiento de los organismos duró $21 \mathrm{~d}$, con mortalidad del $20 \%$, sobreviviendo 36 de los 45 organismos capturados. Durante el proceso de adaptación al consumo de alimento, no se presentó mortalidad en los organismos. Transcurridos los 12 meses de cultivo, se registró un peso promedio final de $720 \pm 50 \mathrm{~g}$ y LF de $31 \pm$ $1 \mathrm{~cm}$ (Figura 1), con tasa de crecimiento en longitud de $0.084 \pm 0.02 \mathrm{~cm} \mathrm{~d}^{-1}$ y peso de $1.97 \pm 0.4$ $\mathrm{g} \mathrm{d}^{-1}$.

En el mes de septiembre los peces se encontraban inmaduros sexualmente, las hembras presentaron ovocitos es estadio de previtelogénesis con diámetro promedio de $190 \pm 22 \mu \mathrm{m}$, mientras que en el mes de noviembre todos los organismos tuvieron el peso y la talla de primera madurez registrada para la especie, que es de 336 y $28 \mathrm{~cm}$, respectivamente. Los ovocitos presentaron el estadio de vitelogénesis con diámetro promedio de $350 \pm 35 \mu \mathrm{m}$, en el mes de diciembre (Figura 2). Mientras que en el mes de enero los peces maduraron sexualmente, en la misma temporada en que lo hacen en el medio natural, observándose ocho hembras con ovocitos en vitelogénesis avanzada y en hidratación con diámetro de 420 a $790 \mu \mathrm{m}$ y 16 machos con semen fluido, mientras que el resto de organismos no presentaron madurez sexual. El peso y talla promedio de los peces inducidos fue de 560 $\pm 50 \mathrm{~g}$ y $30 \pm 1.3 \mathrm{~cm}$. A las $48 \mathrm{~h}$ post-inyección de la hormona gonadotropina coriónica y sérica los reproductores desovaron, por lo que se registró un total de 60000 huevos viables $\mathrm{kg}^{-1}$, con tasa de eclosión del $80 \%$, por medio de desoves parciales cada $24 \mathrm{~h}$ por cinco ocasiones, obteniéndose 35
000 huevos el primer día, 10000 el segundo día y 5000 los siguientes tres días. Los huevos presentaron un diámetro promedio de $797.4 \pm 10.3 \mu \mathrm{m}$, promedio del volumen del saco vitelino de $232 \pm$ $5 \mathrm{~nL}$, diámetro de gota de aceite promedio de 187 $\pm 10 \mu \mathrm{m}$ y LN promedio de los eleuteroembriones al eclosionar de $1.4 \pm 0.16 \mathrm{~mm}$, la supervivencia de las larvas al día siete después de la eclosión fue del $50 \%$ y al día nueve DDE la mortalidad fue del 100\% (Tabla 1).

\section{Desove de organismos silvestres}

Del total de los reproductores silvestres colectados en el mes de febrero, se detectaron dos hembras con peso y LF promedio de $1200 \pm 100 \mathrm{~g}$ y $35 \pm 1.2 \mathrm{~cm}$, respectivamente; con ovocitos en vitelogénesis avanzada de $450 \pm 25 \mu \mathrm{m}$ y cuatro machos con semen fluido, con peso y LF promedio de $1000 \pm 89 \mathrm{~g}$ y $30 \pm 1 \mathrm{~cm}$, respectivamente. Estos peces fueron inducidos con la hormona gonadotropina coriónica y sérica logrando el desove a las $72 \mathrm{~h}$. A partir del desove se obtuvo un total de 40000 huevos $\mathrm{kg}^{-1}$, con desoves parciales cada $24 \mathrm{~h}$ por tres ocasiones, obteniendo 20000 huevos el primer día, y 10000 el segundo y tercer día. Los huevos midieron $838.2 \pm 15.4 \mu \mathrm{m}$, el volumen del saco vitelino fue de $252 \pm 4 \mathrm{~nL}$, el diámetro de la gota de aceite de $220 \pm 7 \mu \mathrm{m}$ y la tasa de eclosión del $60 \%$. En tanto que la LN promedio de los eleuteroembriones fue de $1.9 \pm 0.16 \mathrm{~mm}$, en tanto que la supervivencia de las larvas al día siete después de la eclosión fue del 50\% y al noveno día la mortalidad fue $100 \%$ (Tabla 1 ).

\section{Descripción de eleuteroembriones y larvas}

El análisis de comparación entre grupos con la prueba de $t$ de Student, mostró diferencias significativas $(p<0.05)$, de igual manera el porcentaje de eclosión entre grupos con la prueba de $\mathrm{Chi}^{2}$, registró diferencias significativas. Mientras que el análisis del porcentaje de supervivencia de las larvas entre los grupos, no fue diferente, debido a que se tuvo la misma supervivencia del $50 \%$ al séptimo día después de la eclosión en ambos grupos de larvas (Tabla 1). Los principales cambios morfológicos registra- 


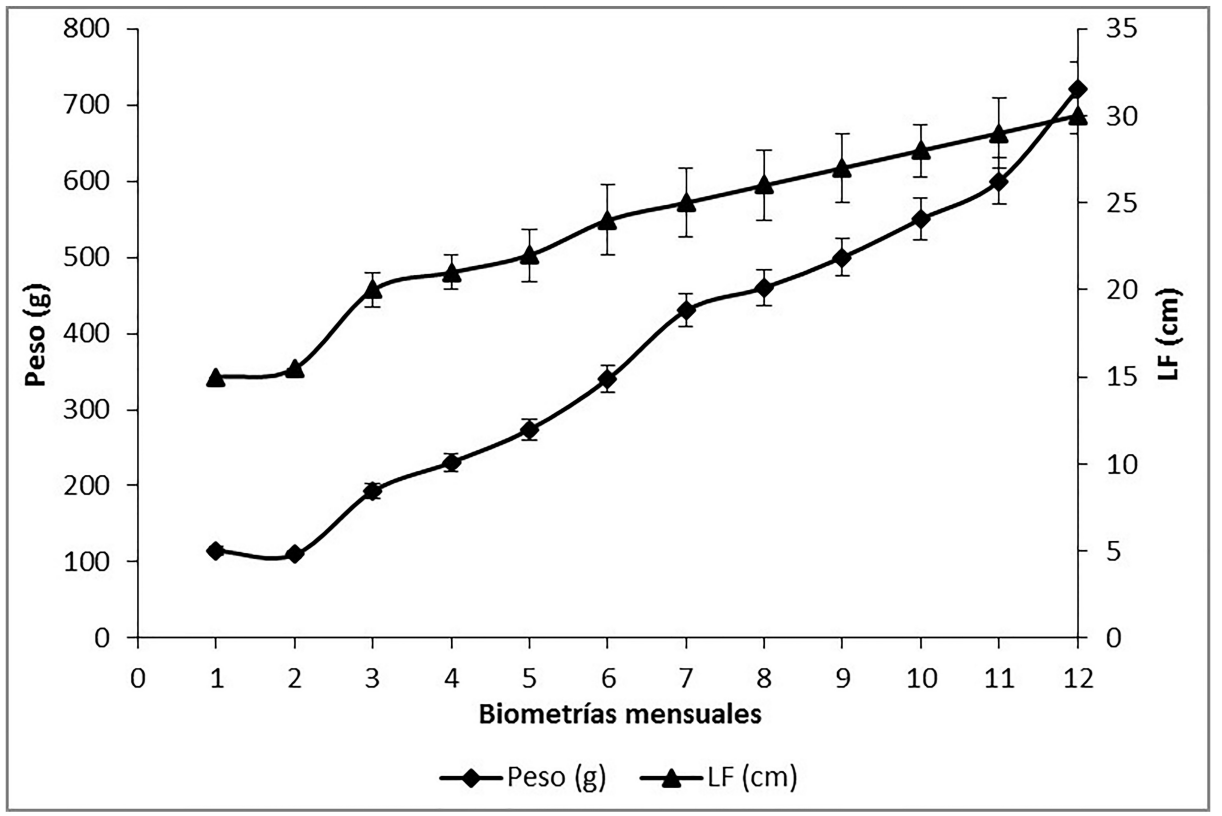

Figura 1. Peso promedio $(\mathrm{g})$ y longitud furcal promedio $(\mathrm{cm})$ registrados en sargos $(A$. probatocephalus) acondicionados al cautiverio por un año.
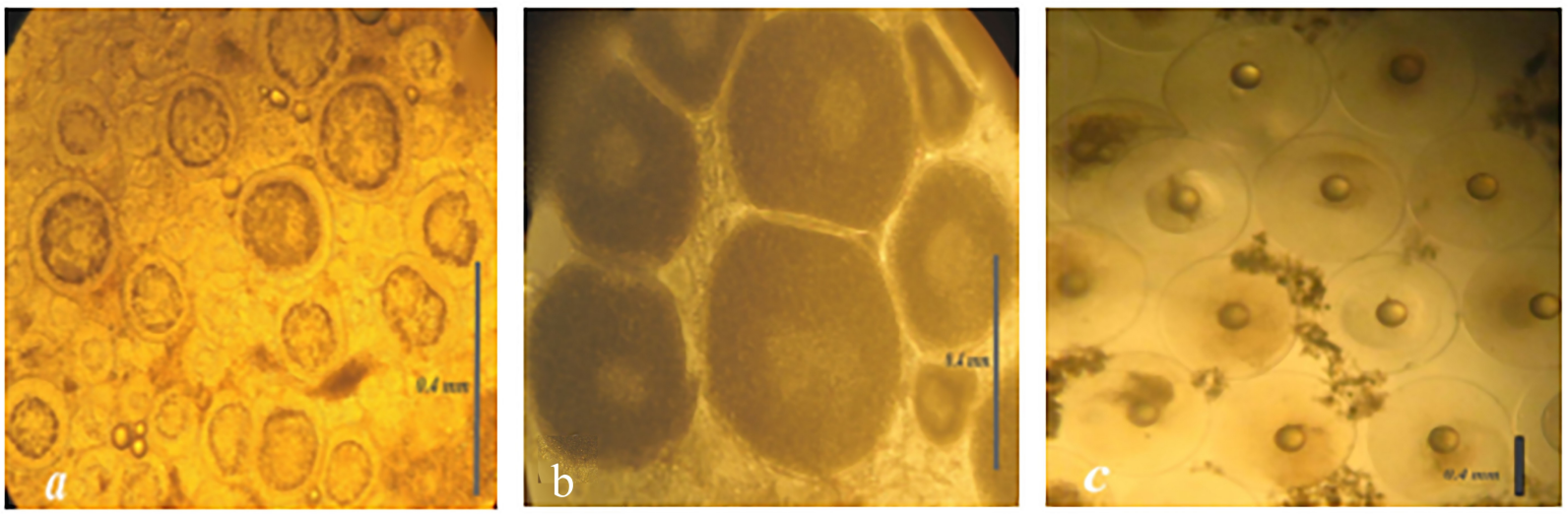

Figura 2. Microfotografías de diferentes estadios de maduración sexual de ovocitos de sargo: (a) estadio previtelogénesis en el mes de septiembre de 2014, con diámetro de $190 \pm 22 \mu \mathrm{m}(\mathrm{n}=30)(10 \mathrm{x})$; (b) estadio de vitelogénesis, en el mes de noviembre de 2014, con diámetro de $350 \pm 35 \mu \mathrm{m}(\mathrm{n}=28)(10 \mathrm{x})$ y $(\mathrm{c})$ estadio de hidratación con diámetro $790 \pm 25 \mu \mathrm{m}$, en el mes de enero de 2015, con la evidente gota de grasa $(n=30)(4 x)$.

dos durante la ontogenia inicial del sargo fueron: el primer DDE los embriones con LN promedio de 1 $650 \pm 25 \mu \mathrm{m}$, tuvieron saco vitelino con volumen promedio de $243.1 \pm 16.5 \mathrm{~nL}$ y gota de aceite con diámetro de $203.5 \pm 16.5 \mu \mathrm{m}$, al día dos DDE se observó en la mayoría de los eleuteroembriones el inicio de la reabsorción del saco vitelino, con LN de 2 $162.5 \pm 43.3 \mu \mathrm{m}$, al tercer DDE la pigmentación de los ojos se completó, las larvas presentan una LN de $2443.8 \pm 51.5 \mu \mathrm{m}$, al cuarto DDE se tuvo la abertura de la boca y ano, comenzando la mayoría de las larvas la alimentación exógena, con LN de 2475 $\pm 20.4 \mu \mathrm{m}$, al quinto DDE se agotó el saco vitelino y la gota de aceite, se observó el desarrollo de aletas pectorales sin radios, las larvas presentan una LN de $2471.9 \pm 41.3 \mu \mathrm{m}$, al sexto DDE se observó 
Merino-Contreras et al.

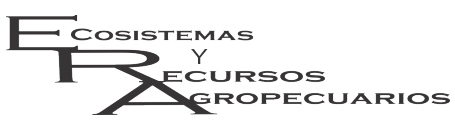

Aclimatación y reproducción de A. probatocephalus

Ecosist. Recur. Agropec.

$5(15): 511-521,2018$

Tabla 1. Características de los desoves, huevos y larvas de sargo ( $A$. probatocephalus) obtenidos de reproductores madurados sexualmente en cautiverio (MC) y silvestres (RS)

\begin{tabular}{|c|c|c|c|}
\hline Característica & Primer desove (MC) & Segundo desove (RS) & Valores promedio \\
\hline Fecha de desove & 6 enero 2015 & 14 febrero 2015 & - \\
\hline Tiempo de desove post-inyección de la hormona & $48 \mathrm{~h}$ & $72 \mathrm{~h}$ & $60 \pm 12 \mathrm{~h}$ \\
\hline Huevos viables & 100000 & 60000 & $80000 \pm 20000$ \\
\hline Temperatura y salinidad de desove & $20.5 \pm 0.5 \mathrm{C}$ y $35 \mathrm{psu}$ & $21.5 \pm 0.5 \mathrm{C}$ y $35 \mathrm{psu}$ & $21 \pm 0.5 \mathrm{C}$ y $35 \mathrm{psu}$ \\
\hline Desoves parciales & 5 & 3 & $4 \pm 1$ \\
\hline Diámetro de los huevos & $796.4 \pm 10.3 \mu \mathrm{m}^{a}$ & $838.2 \pm 15.3 \mu \mathrm{m}^{b}$ & $817.3 \pm 20.9 \mu m$ \\
\hline Tasa de eclosión & $80 \% a$ & $60 \% \mathrm{~b}$ & $70 \%$ \\
\hline Volumen del saco vitelino & $232 \pm 7.8 \mathrm{~nL}^{a}$ & $254.2 \pm 7.2 \mathrm{~nL}^{b}$ & $243 \pm 11 \mathrm{~nL}$ \\
\hline Diámetro de la gota de aceite & $187 \pm 15.4 \mu \mathrm{m}^{a}$ & $220 \pm 7.1 \mu \mathrm{m}^{b}$ & $203.5 \pm 16.5 \mu m$ \\
\hline Longitud notocordal de eleuteroembriones & $1.4 \pm 0.16 \mathrm{~mm}^{a}$ & $1.9 \pm 0.1 \mathrm{~mm}^{b}$ & $1.65 \pm 0.25 \mathrm{~mm}$ \\
\hline Supervivencia de larvas al séptimo DDE & $50 \%^{a}$ & $50 \%^{a}$ & $50 \%$ \\
\hline
\end{tabular}

el inicio del patrón de pigmentación característico de la especie con la presencia de melanóforos dendríticos en cabeza, ano y línea ventral, LN de 2 $568.8 \pm 65.7 \mu \mathrm{m}$, en el séptimo y octavo DDE no se observaron cambios aparentes, solo incremento en longitud, al noveno DDE, algunas larvas inician el estadio de preflexión, con la aparición de aletas pectorales con radios, y $\mathrm{LN}$ de $2741.3 \pm 23.9 \mu \mathrm{m}$ (Tabla 2 y Figura 3 ).

\section{DISCUSIÓN}

El lote de peces acondicionados en cautiverio tuvo a los 12 meses un peso promedio de $720 \pm 50 \mathrm{~g}$, peso mayor al registrado por Tucker $(1998,2004)$, quien reportó un peso promedio de $480 \mathrm{~g}$ en 12 meses de cultivo. Se tuvo un incremento en peso de $1.97 \pm 0.4 \mathrm{~g} \mathrm{~d}^{-1}$, que es similar a lo reportado por Tucker (2004), cuando alimentó un lote de sargos con alimento comercial de trucha. Hay registros de que la tasa de crecimiento de los peces se modifica por factores como la temperatura, la densidad de organismos, el porcentaje de alimentación y el tipo de alimento (Lahti y Lower 2000, Wang et al. 2000). El sargo alcanzó mayor tamaño en el mismo periodo de tiempo, debido probablemente a la naturaleza de la dieta suministrada, así como al efecto de la temperatura promedio anual de la zona de estudio, la cual es mayor que la registrada por Tucker (1998).

La maduración sexual y desove de los organismos en cautiverio ocurre en el mes de enero, y en el lote de organismos silvestres en el mes de febrero, lo que coincide con lo reportado por Mc Donough et al. (2011) quienes señalan que esta especie se reproduce de enero a abril. La mayoría de los organismos maduraron sexualmente al año de su aclimatación en cautiverio, a diferencia de lo reportado por Tucker $(1998,2004)$ y Wenner (2004), quienes señalan que lo hacen a los dos años. Mientras que Tucker y Barbera (1987), obtuvieron la madurez sexual, de algunos machos de un lote de sargos en cautiverio por un año, en condiciones de cultivo similares a las del presente estudio. Lo que se puede deber a que la dieta suministrada, cubrió los requerimientos nutricionales de los reproductores, lo que permitió la maduración sexual y desove, debido a que contiene ingredientes, reconocidos como componentes importantes en dietas de maduración empleadas en varias especies de peces marinos (Watanabe y Vassallo-Agius 2003). Por lo que se considera que estos ingredientes influyeron en la fecundidad de los peces, en la cantidad de huevos viables, en la reducción de anormalidades en huevos y embriones, así como en la tasa de eclosión, por los beneficios de su composición en ácidos grasos, nivel proteico, buena digestibilidad y a su contenido de fosfolípidos y colesterol (Fernández-Palacios et al. 1997, Watanabe y Vassallo-Agius 2003).

Al comparar las dosis empleadas de la hormona gonadotropina coriónica humana $(\mathrm{HGCH})$, por otros autores para inducir al desove de otras especies de peces de la familia Sparidae, la hormona 
Tabla 2. Longitud de la notocorda (LN) registrada durante la ontogenia inicial de sargo ( $A$. probatocephalus), día después de la eclosión (DEE), N (tamaño de la muestra).

\begin{tabular}{|c|c|c|c|}
\hline Día & $\mathrm{N}$ & $\mathrm{LN}(\mu \mathrm{m})$ & Evento \\
\hline 0 & 30 & $817.3 \pm 20.9$ & Desove \\
\hline 1 & 30 & $1650 \pm 25$ & Eclosión \\
\hline 2 & 20 & $2162.5 \pm 43.3$ & Inicia reabsorción de saco vitelino \\
\hline 3 & 20 & $2443.8 \pm 51.5$ & Se completa la pigmentación de los ojos \\
\hline 4 & 20 & $2475.0 \pm 20.4$ & Abertura de boca y ano, comienza alimentación exógena \\
\hline 5 & 15 & $2471.9 \pm 41.3$ & Agotamiento de saco vitelino y gota de aceite, desarrollo de aletas pectorales sin radios \\
\hline 6 & 15 & $2568.8 \pm 65.7$ & Melanóforos dendríticos visibles en cabeza, ano y línea ventral, que marcan la pigmentación específica \\
\hline 9 & 15 & $2741.3 \pm 23.9$ & Inicia preflexión, aletas pectorales con radios \\
\hline
\end{tabular}

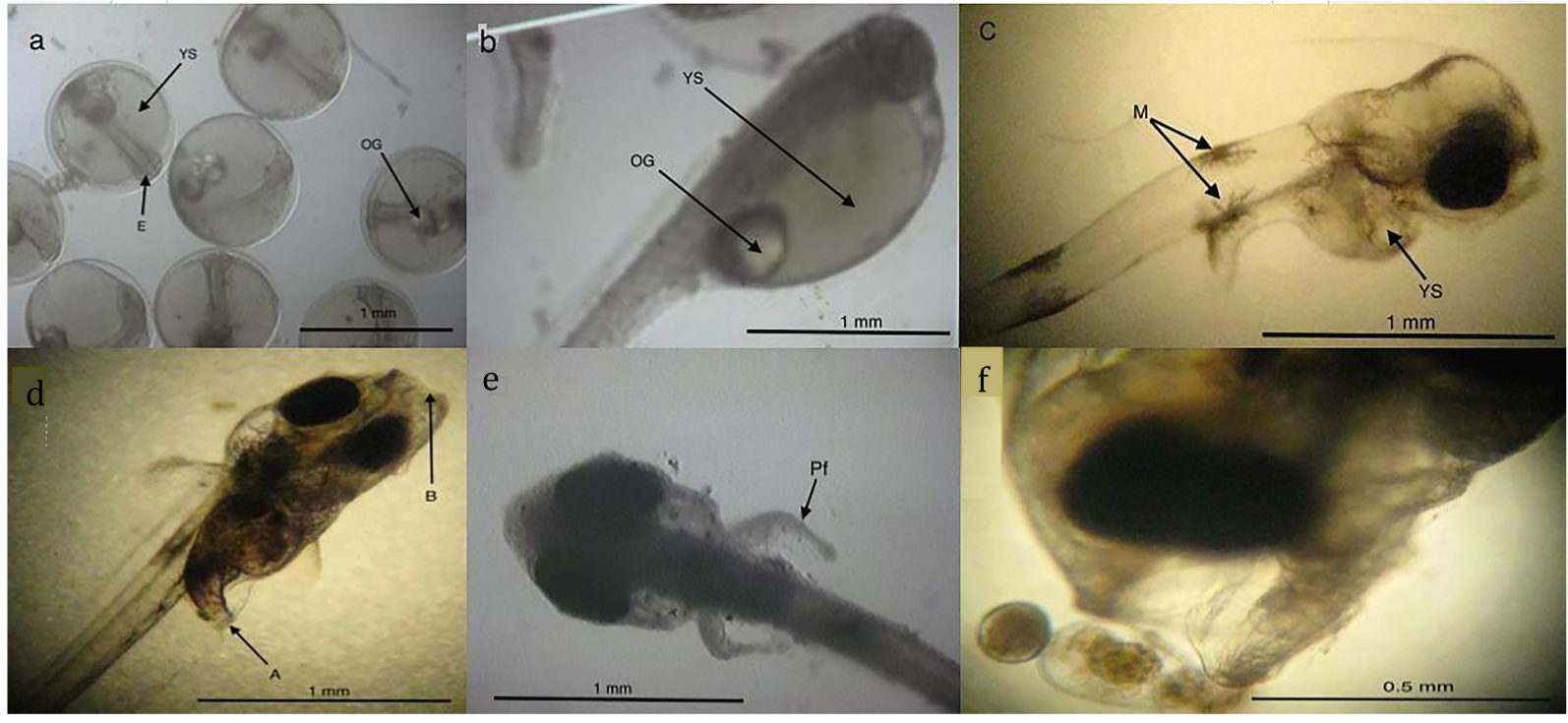

Figura 3. Principales cambios morfológicos registrados en la ontogenia inicial de larvas de sargo (A. probatocephalus): (a) Huevos antes de la eclosión, se observa el embrión (E), el saco vitelino (YS) y la única gota de aceite (OG); (b) eleuteroembrión recién eclosionado (1 DDE), se señala el prominente saco vitelino (YS) y la gota de aceite (OG); (c) larva de 3 DDE, se observa la reabsorción parcial de saco vitelino (YS) y melanóforos dendríticos visibles (M); (d) larva de 4 DDE con boca (M) y ano (A) abiertos; (e) larva en preflexión a los 9 DDE, se observan las aletas pélvicas con radios (Pf); (f) parte anterior de larva y rotífero $(R)$, microfotografías $4 x$.

homóloga de la gonadotropina coriónica y sérica empleada en el presente estudio, varía de 100 a $250 \mathrm{UI} \mathrm{kg}{ }^{-1}$ en la dorada Sparus aurata (Colombo et al. 1989), hasta 1000 a $2000 \mathrm{Ul} \mathrm{kg}^{-1}$ en el sargo (Tucker y Barbera 1987, Tucker 1998), quienes sugieren dos aplicaciones vía intramuscular de $1000 \mathrm{UI} \mathrm{kg}{ }^{-1}$, separadas de 24 a $36 \mathrm{~h}$, entre cada aplicación, logrando la ovulación de 24 a 76 $h$ después de la inyección de la hormona. La inducción al desove en ambos lotes de sargos en el presente estudio, con $500 \mathrm{UI} \mathrm{kg}^{-1}$ de la hormona gonadotropina coriónica y sérica a dosis menor que la recomendada, indujo dos desoves. Mientras que los peces acondicionados al cautiverio desovaron a las $48 \mathrm{~h}$ posteriores a la inyección y los peces silvestres a las $76 \mathrm{~h}$, diferencias que se pueden deber al estrés provocado en la captura, traslado y manejo en los organismos silvestres.

El promedio del diámetro del ovocito maduro fue de $420 \mu \mathrm{m} \pm 35$, valores que son ligeramente mayores a lo reportado por Tucker y Barbera (1987). Lo que indujo al desove y producción de huevos viables, con mayor frecuencia de desoves parciales (de cuatro a cinco) post-inyección, que los reportados por Tucker $(1998,2004)$. Pero con menor cantidad de huevos viables y de desoves parciales en el 


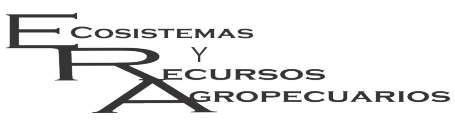

desove de los organismos silvestres, que en el lote de organismos acondicionados al cautiverio, lo que se puede deber al estrés de captura y traslado, ya que se tienen registros que señalan, que dicha condición puede provocar en las hembras un retardo en la maduración del ovocito, vitelogénesis incompleta, inhibición de la ovulación y puesta, lo que causa atresia folicular acelerada y en los machos inhibe la producción d e esperma y disminuye la fluidez del semen (Goos y Casten 2002, Castranova et al. 2005). Estos efectos e intensidad pueden variar con la especie, el estado de maduración y el grado de tolerancia al estrés (Schreck et al. 2001).

La calidad de los huevos en el desove es la habilidad del huevo para fertilizarse y desarrollar un embrión normal (Bobe y Labbé 2010). Aunque las tasas de fertilización y eclosión son los indicadores más utilizados en la evaluación de los desoves, otros parámetros como la morfología del huevo, diámetro, distribución de sus gotas de aceite, anormalidad de los blastómeros, tamaño de las larvas, volumen del saco vitelino y deformidad larval, también pueden considerarse indicadores confiables de la evaluación (Lahnsteiner y Patarnello 2005, Rideout et al. 2005). En ambos desoves del presente estudio se consideran de buena calidad, en base a los criterios descritos con anterioridad. Los huevos y larvas obtenidos del desove de los reproductores silvestres tuvieron mayor tamaño que los de los peces acondicionados en cautiverio; con tallas promedio registradas son similares a las reportado por Tucker y Alshuth (1997). Esta diferencia puede deberse a que, en los peces acondicionados a cautiverio, era su primer desove, y para los peces silvestres por las tallas y pesos que presentaron posiblemente fuera su segunda o tercera época de reproducción, al respecto se reporta relación positiva entre el tamaño y la edad de las hembras y sus huevos, así como con la calidad del desove (Faulk y Holt 2008, Quintero et al. 2009). Las mejores tasas de fecundación, eclosión y tamaño de huevos, se tiene con hembras de mediana edad (Trippel 1998, Laine y Rajasilta 1999). En algunas especies se ha demostrado que el diámetro del huevo aumenta con la edad de los reproductores, por lo que, huevos más grandes, pro-
Aclimatación y reproducción de A. probatocephalus

Ecosist. Recur. Agropec.

5(15):511-521,2018

producen por lo general larvas más grandes, con mayor cantidad de nutrientes de reserva (Kennedy et al. 2007), como lo observado en el desove de los organismos silvestres.

La mayor mortalidad en las larvas de peces marinos se presenta durante los primeros días de vida (Tucker 1998, Álvarez-Lajonchére y Hernández 1991), lo que tiene que ver con el tamaño del alimento vivo suministrado y con el valor nutricional del mismo. La eficiencia alimenticia de las larvas es afectada por factores bióticos como la densidad de la presa y su distribución, el tamaño, la movilidad, el color, el tamaño del depredador, el tamaño de la boca, así como el grado de desarrollo del sistema visual y del tubo digestivo (Seiffer et al. 2001, ZavalaLeal et al. 2013). El efecto de estos factores en la sobrevivencia, indica que el tamaño de la presa y el tamaño de la boca de las larvas, fueron los que más influyeron, ya que aunque, tradicionalmente las larvas de peces marinos son alimentadas inicialmente con el rotífero Brachionus plicatilis morfotipo So pequeño (Prieto et al. 2006), en el presente estudio, fueron alimentadas con el morfotipo $L$ (232-300 $\mu \mathrm{m})$, que es más grande que la boca de las larvas de sargo, lo que provocó que su supervivencia al séptimo DDE fuera del $50 \%$, y al día nueve DDE las larvas de ambos desoves, presentaron una mortalidad del $100 \%$, por inanición. Se desconocía al momento de administrar el primer alimento vivo el tamaño de la boca de las larvas, por lo que se dio por sentado que podrían alimentarse de forma adecuada con el rotífero suministrado, lo que no ocurrió. Al respecto Tucker y Barbera (1987) reportaron una supervivencia al séptimo DDE del $62 \%$ y Tucker y Alshuth (1997) del $40 \%$ a los $10 \mathrm{DDE}$, alimentando larvas de sargo con $B$. plicatilis morfotipo $S$, por lo que se tiene que revisar el esquema de alimentación para la obtención de mejores resultados en la supervivencia de las larvas.

\section{CONCLUSIONES}

El lote de juveniles se acondicionó al cautiverio, maduró sexualmente y se reprodujo, tanto el lote de organismos acondicionados como silvestres 
respondieron de forma satisfactoria a la inducción al desove con la hormona gonadotropina coriónica y sérica; con dos desoves con buenos resultados. Los huevos y larvas de los reproductores silvestres presentaron mayor talla; pero las tasas de sobrevivencia fueron iguales para ambos lotes de larvas. Fue posible la reproducción en cautiverio de esta especie, lo que permitirá la producción de crías para su futuro aprovechamiento.

\section{AGRADECIMIENTOS}

Al Tecnológico Nacional de México por el apoyo económico otorgado para la realización del presente trabajo con el proyecto de investigación titulado: Fisiología digestiva de larvas de sargo Archosargus probatocephalus (Perciformes: Sparidae), clave 5247.14.

\section{LITERATURA CITADA}

APROMAR (2017) La Acuicultura en España, 2017. APROMAR Unión Europea, Gobierno de España, 93 p. http://www.apromar.es/sites/default/files/2017/informe/APROMAR_Informe_ACUICULTURA 2017, pdf. Fecha de consulta: 6 de diciembre 2017.

Álvarez-Lajonchére L, Hernández O (1991) Producción de juveniles de peces estuarinos para un centro de América Latina y el Caribe: Diseño, Operación y Tecnología. Word Aquaculture Society, Baton Rouge USA, 424p.

Bester y Robins (2005) Biological profiles: sheepshead. Ichthyology: Education-Biological profiles. Florida museum of natural history University of Florida www.flmnh.ufl.edu/fish/Gallery/Descript/Sheepshead/ Sheepshead.html. Fecha de consulta: 15 de junio 2017.

Bobe J, Labbé C (2010) Egg and sperm quality in fish. General and Comparative Endocrinology 165: 535-548.

Castranova, DA, King W, Woods LC III (2005) The effects of stress on androgen production, spermiation response and sperm quality in high and low cortisol responsive domesticated male striped bass. Aquaculture 246: 413-422.

Colombo L, Francescon A, Barbaro A, Belvedere P, Melotti P (1989) Induction of spawning in the Gilthead sea bream, Sparus aurata L., by elevation of the water temperature and salinity and by HCG LH-RH analogue treatments. Rivista Italiana di Acquacoltura 24: 187-196.

Faulk CK, Holt, GJ (2008) Biochemical composition and quality of captive spawned cobia Rachycentron canadum eggs. Aquaculture 279: 70-76.

Fernandez-Palacios H, Izquierdo M, Robaina L, Valencia A, Salhi M, Montero D (1997) The effect of dietary protein and lipid from squid and fish meals on egg quality of broostock for gilthead seabream (Sparus aurata). Aquaculture 148: 233-246.

GSMFC (2006) The sheepshead fisheries, pub. No. 143 of The Gulf of Mexico, United States. A Fisheries profile, by The sheepshead technical task force. Mississippi, EUA. 143p.

Goos HJ, Costen D (2002) Stress adaptation, cortisol and pubertal development in the male common carp, Cyprinus carpio. Molecular and Cellular Endocrinology 197: 105-116.

Kennedy J, Geffen AJ, Nash RDM (2007) Maternal influences on egg and larval characteristics of plaice (Pleuronectes platessa L.). Journal of Sea Research 58: 65-77.

Lahnsteiner F, Patarnello P (2005) The shape of the lipid vesicle is a potential marker for egg quality determination in the gilthead seabream, Sparus aurata, and in the sharpsnout seabream, Diplodus puntazzo. Aquaculture 246: 423-435. 
Lahti K, Lower N (2000) Effects of size asymmety on agression and food acquisition in Arctic charr. Journal of fish Biology 56: 915-922.

Laine P, Rajasilta M (1999) The hatching success of Baltic herring eggs and its relation to female condition. Journal of Experimental Marine Biology and Ecology 237: 61-73.

McDonough CJ, Wenner CA, Roumillat WA (2011) Age, Growth, and Reproduction of Sheepsheads in South Carolina. Marine and Coastal Fisheries 3: 366-382.

Munyandorero J, Murphy MD, MacDonald TC (2006) An assessment of the status of sheepshead in Florida waters through 2004. Florida Fish and Wildlife Conservation Commission, Florida Marine Research, Florida EUA 113p.

Okuzawa K, Takebe T, Hirai N, Ikuta K (2015) Status of resource enhancement and sustainable aquaculture practices in Japan. In: Romana-Eguia MRR, Parado-Estepa FD, Salayo ND, Lebata-Ramos MJH (Eds.) Resource enhancement and sustainable aquaculture practices in southeast Asia: Challenges in responsible production of aquatic species. Aquaculture Dept., Southeast Asian Fisheries Development Center. Tigbauan Iloilo, Philippines. pp: 41-52.

Prieto M, Castaño F, Sierra J, Logato P, Botero J (2006) Alimento vivo en la larvicultura de peces marinos: copépodos y mesocosmos. Revista MVZ Córdoba 11: 30-36.

Quintero HE, Durland E, Davis DA, Dunham R (2009) Effect of lipid supplementation on reproductive performance of female channel catfish, Ictalurus punctatus, induced and strip-spawned for hybridization. Aquaculture Nutrition 17: 117-129.

Rideout RM, Rose GA, Burton MPM (2005) Skipped spawning in female iteroparous fishes. Fish and Fisheries 6: $50-72$

Schreck CB, Contreras-Sánchez W, Fitzpatrick MS (2001) Effects of stress on fish reproduction gamete quality and progeny. Aquaculture 197: 3-24.

Seiffert B (2001) Effect of dietary (n-3) highly unsaturated fatty acids on growth and survival of fat snook (Centropomus parallelus, Pisces: Centropomidae) larvae during fish feeding. Brazilian Journal Medical Biological Research 34: 645-651.

Trippel EA (1998) Egg size and viability and seasonal offspring production of young Atlantic cod. Transactions of the American Fisheries Society 127: 339-359.

Tucker JW Jr (1998) Marine fish culture. Kluwer Academic Publishers, Massachusetts, USA. 750 p.

Tucker, JW Jr (2004) Sheepshead, a potential American sea bream for farming. World Aquaculture Magazine 35: $48-68$.

Tucker JW Jr, Alshuth SR (1997) Development of laboratory-reared sheepshead, Archosargus probatocephalus (Pisces: Sparidae). Fishery Bulletin 95: 394-401.

Tucker JW Jr, Barbera PA (1987) Laboratory of spawning of shepshaed. The Progresive Fish-Culturist 49: 229-230.

Wang N, Hayward RS, Noltie DB (2000) Effects of social interaction on growth of juvenile hybrid sunfish held at two densities. North American Journal of Aquaculture 62: 161-167.

Watanabe T, Vassello-Agius R (2003) Broodstock nutrition research on marine finfish in Japan. Aquaculture 227: $35-61$. 


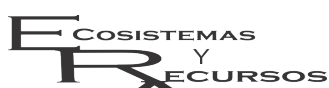

$\sum_{\text {ZCursos }}$

GROPECUARIOS
Aclimatación y reproducción de A. probatocephalus

Ecosist. Recur. Agropec. 5(15):511-521,2018

Wenner C (2004) Status and trends in the fishery and population of sheepshead in South Carolina waters. Technical Report to the South Carolina Department of Natural Resources. Marine Resources Division Publications Carolina, USA. 64p.

Zavala-Leal I, Dumas S, López-Villegas EO, Peña E, Contreras-Olguín M, Flores-Montijo L, et al. (2013) Structural development of Pacific red snapper Lutjanus peru from hatching to the onset of first feeding. Aquaculture Research 45: 519-527. 
